\title{
Three methods for estimation of changes in frequency characteristics of potentials elicited by long-lasting (fatiguing) activity of isolated muscle fibres
}

\author{
Teodora I. Vukova, Vladimir Dimitrov and Nikolina Radicheva \\ Institute of Biophysics, Bulgarian Academy of Sciences, Acad. G. Bontchev Str. Bl. 21, Sofia, Bulgaria
}

\begin{abstract}
The present study was assigned to compare the applicability and sensitivity of three different methods (discrete wavelet transforms (DWT), median frequency (MDF) - calculated on the basis of fast Fourier transform, and spectral indices) for analysis of frequency content changes in potentials produced by repetitive stimulation from isolated slow (SMFs)- and fast (FMFs)-fatigable muscle fibers during uninterrupted activity. In order to affect fatigue, prior to starting the fatiguing stimulation $(5 \mathrm{~Hz})$, some of the fibres were exposed to microwave electromagnetic field and others were sham exposed. All the methods studied demonstrated that during a long-lasting fibre activity, the shift of the potential frequency content to lower frequencies was a consequence of lowering in higher frequency components and increasing in low frequency ones. The changes were faster in FMFs than SMFs and stronger in sham exposed fibres $v s$. microwave exposed. The MDF alterations in both fibre types and protocols were quite similar to other methods characterizing either individual changes in low and high frequency scales of the signal (discrete wavelet transform - DWT) or changes in their spectral ratio (spectral indices). The spectral indices showed sensitivity to fatigue effect at the final stages that made their changes considerably non-linear. DWT allowed processing the temporal occurrence of frequency content changes that corresponded to the alterations in the separate potential phases as well as earlier detection and precise evaluation of fatigue onset and progressing.

All methods are applicable but DWT is a preferable for study of muscle fibre fatigue in clinical and experimental neurophysiology.
\end{abstract}

Key words: Peripheral muscle fatigue - Muscle fibre types - Wavelet discrete transform — Spectral indices - Microwave electromagnetic field

\begin{abstract}
Abbreviations: E-C, excitation-contraction coupling; PV, propagation velocity; EMG, electromyography; ECAP, extracellular action potential; ICAP, intracellular action potential; FFT, Fast Fourier transform; MDF, median frequency; SFT, short-time Fourier transform; DWT, discrete wavelet transform; MMW, microwave; SMF, slow-fatigable muscle fibre; FMF, fast-fatigable muscle fibre; ET, endurance time; RMS, root mean squire.
\end{abstract}

\section{Introduction}

Fatigue is a process of a changed functional state of the continuously stimulated muscle. The mechanisms un-

Correspondence to: Teodora I. Vukova, Institute of Biophysics, Bulgarian Academy of Sciences, Acad. G. Bontchev Str., Bl. 21, Sofia 1113, Bulgaria

E-mail: tivukova@.bio.bas.bg derlying peripheral fatigue may differ depending on the muscle (Bigland-Ritchie et al. 1978) or its fibre composition (Colliander et al. 1988). During repetitive stimulation, diminished membrane excitability predetermines a failure of excitation-contraction coupling (E-C) (Eberstein and Sandow 1963; Grabowski et al. 1972). Warren et al. (1999) report that eccentric contraction may lead to E-C coupling failure, whose site lies below the plasmolemma and above the sarcoplasmic reticulum $\mathrm{Ca}^{2+}$-release channels. Fatigue- 
induced changes in membrane properties lead to an action potentials' decrease of amplitude and propagation velocity (PV) of excitation, an increase of time-domain parameters (Jones 1981; Metzger and Fitts 1986; Radicheva et al. 1986; Mileva et al. 1998) and shifting of the spectral characteristics to the low frequency range (Lindström et al. 1970; Dolan et al. 1995). Some authors consider that the frequency shift is questionable (Vøllestad 1997) because fatigue is not always accompanied by significant changes of electromyographic (EMG) spectral characteristics (Moxham et al. 1982). Thus, it is not unnecessary more investigations about this problem to be done for looking up different approaches and new methods for frequency analysis which could catch the most slight and earliest alterations in that characteristics of the muscle potentials.

The changes in frequency content of the potentials depend on potential parameters which are related to the method of their obtaining: intracellular or extracellular. The position of the recording electrode in radial direction, as well as the position along the fibre i.e. close to the stimulating electrode, to the fibre conical part, over fibre end or beyond the end as the PV of excitation are of importance. In the present study we analyzed potentials recorded close (up to $50 \mu \mathrm{m}$ ) to the membrane over the middle part of the fibre. These parameters of the record determine the shape of the extracellular action potential (ECAP). It is known that extracellular potential reflects the shape of the intracellular action potential (ICAP) which, on the other hand, is closely related to the membrane ionic processes. The changed membrane excitability by different factors, for example fatigue, leads to changes in the characteristics of ICAP and hence in ECAP. The space derivatives of the ICAP are also closely related to ECAP characteristics (Radicheva et al. 1986). Their parameters (to be more precise $\mathrm{T}_{1}$ - time interval between positive and negative maxima) are strongly influenced by the changes in the front (depolarization phase duration named rising time) of ICAP (Radicheva 1986a,b). It was also known that the shape of potentials recorded far from the fibre membrane $(3000 \mu \mathrm{m})$ depend on the ICAP shape as well as on PV of excitation (Radicheva and Kolev 1992; Radicheva et al. 1993). Therefore the average ECAPs recorded at large radial distance from the membrane can be viewed as motor unit potentials recorded by superficial EMG electrodes (Gydikov et al. 1986a,b; Gydikov 1992; Radicheva et al. 1993). Model studies with different protocols show the same results (Slavcheva et al. 1996; Dimitrova and Dimitrov 2006; Arabadzhiev et al. 2008). Just because of this reason we consider that the obtained information by our experimental model for peripheral muscle fatigue estimated by changes in frequency characteristics of the potentials would be important for clinical neurophysiology. All these statements substantial for potentials from isolated muscle fibres also concern EMG signals recorded by superficial or penetrat- ing - wire or needle electrodes. This assumes that under the latter condition when the electrode is close to the active fibre membrane, the obtained potentials are similar to the potentials analyzed in the present study and the results concern both group potentials - "in vitro" as "in situ" recorded.

The changes in power spectrum of different biological signals (EMG, EEG, intra- and extracellular potentials from single muscle fibers, etc.) are usually evaluated by Fast Fourier transforms - FFT (Sprato et al. 1999; Pope et al. 2000; Vydevska-Chichova et al. 2007). On the basis of FFT calculated parameters are median frequency (MDF) and mean frequency. MDF is widely used to estimate the changes in the frequency content of EMG potentials with fatigue development because MDF reflects the total power spectrum. FFT is usually applied on signals with stationary distribution of frequencies in time. To avoid this problems of Fourier transforms, the signals can be divided into blocks with short durations and thus FFT is modified as short-time Fourier transform (SFT). This method, however, has restrictions due to a time-frequency resolution - time and frequency resolutions are fixed (Hostens et al. 2004; Beck et al. 2005) which can be compensated by other methods (Cohen's class time-frequency distributions as well as wavelet analysis).

The discrete wavelet transform (DWT) became a widespread tool for analyzing localized variations of power spectra within time-frequency variation (Daubechies 1992; Morlet et al. 1993; Torrence and Compo 1998; Sprato et al. 2000; Wang et al. 2004). DWT as a method of multi-resolution analysis has been used successfully for processing the occurrence of frequency alterations in biological signals such as EMG of compound potentials (Daubechies 1992; Coorevits et al. 2008), EEG (Subasi et al. 2005), ECG (Froesea et al. 2006) caused by different physical and chemical influences.

The advantage of wavelets over SFT, is that wavelet transforms vary in time-frequency ratio, producing good frequency localization at low frequencies (long time windows) and good time localization at high frequencies (short time windows) (Mallat 1989; Karlsson et al. 1999; Karlsson et al. 2000; Hostens et al. 2004).

Arabadzhiev et al. (2005) paid attention to the effect of increase of ICAP duration and the negative after-potentials on power spectra of surface detected EMG signals and proposed a new method for frequency analysis called spectral indices whose sensitivity to muscle fatigue was higher than that of MDF (Dimitrova et al. 2005). These indices have been tested on M-waves from muscle biceps brachii during repetitive activity slightly above the threshold electrical stimulation (Arabadzhiev et al. 2005; Dimitrova et al. 2009). The same indices were successfully applied in experimental examinations assessing peripheral muscle fatigue (Dimitrov et al. 2006; Dimitrov 2008). Comparing the sensitivity and applicability of mean and MDF and the four spectral indices during dynamic contractions, good reliability was observed 
of these methods for frequency analysis but the sensitivity of the spectral indices was much more pronounced (Dimitrov et al. 2006).

To the best of our knowledge in the available literature there is no method approved as the most appropriate for spectral analysis of single muscle fibre potentials. We carried out preliminary investigations (Vukova et al. 2008) with that goal comparing applicability of DWT and FFT. The results obtained showed that DWT is a preferable method. But in the last few years the new method for spectral analysis (spectral indices) calculated as a ratio between low and high frequency potential content found the approval of many authors for their better accuracy and sensitivity for examination of fatigue-induced changes of superficially recorded EMG signals (motor unit potentials, M-wave) compared to FFT. We decided to apply this new method for analysis of our data.

Hence, the goals of the present work were: 1) to apply new Spectral indices, DWT and FFT for analysis of the fatigueinduced changes in the spectral characteristics of ECAPs obtained close to the repetitively stimulated isolated slow and fast-fatigable frog muscle fibers; 2) to use different approaches for development of fatigue with some distinctions in the stage of induced changes in frequency characteristics of the potentials. Thus we aimed to consider which one of the methods used is the most appropriate to estimate the onset and development of fatigue; and 3) to suggest a preferable methods to the clinical and experimental neurophysiology for frequency analysis of EMG potentials from single muscle fibres.

\section{Materials and Methods}

\section{Experimental protocol}

Small bundles of muscle fibers were dissected from gastrocnemius muscle of Rana ridibunda. The preparations were mounted horizontally in double-wall chamber with temperature controlled bath solution $\left(20-21^{\circ} \mathrm{C}\right)$ between force-transducer and a hook fixed to the opposite side of the chamber. The experiments were carried out at optimal fiber length (length at which the suprathreshold stimulation pulse evoked maximal twitch). During fatiguing protocol that lasted $180 \mathrm{~s}$ the muscle fiber activity was provoked by suprathreshold rectangular pulses (0.5-1 ms duration) with interstimulus interval of $200 \mathrm{~ms}$ (stimulation frequency of $5 \mathrm{~Hz}$ ). The stimulation was applied via a functional generator (Anapulse stimulator, model 305-1, WP Instruments Inc.) with stimulus isolation unit (model 302-T, WP Instruments Inc.). ECAPs were recorded by a pair of glass coated tungsten electrodes with a fixed interelectrode distance of $340 \mu \mathrm{m}$. It was oriented longitudinally to the fibre axis and situated close (up to $50 \mu \mathrm{m}$ ) to the fibre membrane and far from the fibre end and place of the stimulation electrode. The tip diameter of each electrode was $10 \mu \mathrm{m}$ with a resistance of $150 \mathrm{k} \Omega$. The ECAPs were amplified through High performance bioamplifier $(20 \mathrm{kHz}$ bandwidth, Coulbourn Instrumants) and transferred on-line to a computer via an analogue-to-digital converter (CED 1401, Cambridge UK) with a sampling frequency of $12.5 \mathrm{kHz}$ per channel and an accuracy of sampling of 12 bits.

In order to affect the fatigability presented by distinctions in the stages of manifestation of fatigue appearance and development two approaches were applied: a) fatiguing protocol with 3-min trial of repetitive stimulation preceded from an exposure of the fibres for $60 \mathrm{~min}$ on open area. This group of fibres was called control or "sham exposed"; b) fatiguing protocol also with 3-min trial of repetitive stimulation preceded from an exposure to microwave (MMW) $2.45 \mathrm{GHz}$ electromagnetic field with power density of $20 \mathrm{~mW} / \mathrm{cm}^{2}$ of the fibres for $60 \mathrm{~min}$ on open area. This group of fibres was named "MMW exposed". The specific absorption rate of the MMW exposed preparation was $0.06 \mathrm{~mW} / \mathrm{mg}$. The experimental procedure and method for specific absorption rate calculation were studied earlier (Radicheva et al. 2001, 2002). Commonly 54 isolated muscle fibres were used, as 30 fibres were SMFs at 15 for each group (sham exposed and MMW exposed) and 24 fibres - FMFs divided at 12 for both groups. The fatiguing protocol was performed ones for each fibre in both groups (sham exposed and MMW exposed ones) which makes 54 times for both fibre types and groups.

\section{Data analysis}

The pair electrode used for potential registration allows the propagation velocity of excitation to be calculated. The rate of PV changes during the uninterrupted muscle fibre activity was used as a criterion to define the fibre types. The investigated fibres were classified conditionally in two groups - SMFs and FMFs using Cluster analysis (Radicheva et al. 1998).

Endurance time (ET) was defined for each fibre as the time from the first potential produced by the repetitive stimulation to the time point of first potential failure, i.e. ET presented the time period of uninterrupted fibre activity. The potentials of each fibre recorded in this time period were analyzed. To combine results obtained from different fibres, the time period of uninterrupted fibre activity for each fibre was assumed to be $100 \%$ of ET and was divided into 4 equal intervals corresponding to 25, 50, 75 and $100 \%$ of ET.

The ECAPs used for analysis were extracted as follows: 10 consecutive ECAPs (i.e. the potentials in 2-s time epoch) at the chosen four ET intervals were averaged in order to diminish the noise in the records. 
The values of the characteristic time interval between the positive and negative maxima $\left(\mathrm{T}_{1}\right)$ of the extracted ECAPs in both experimental protocols were also measured. Off-line analysis of that data was performed using custom-written scripts developed in Spike2 (CED, Cambridge, UK). The normalized changes in $\mathrm{T}_{1}$ duration were calculated as follows:

$$
\Delta T_{1}[\%]=\frac{T_{1 i}-T_{1}}{T_{1}} \cdot 100
$$

where $T_{1}$ and $T_{1 i}$ is peak-to-peak

time interval for the first and last ECAP at the corresponding, $\mathrm{i}^{\text {th }}$, point interval of ET.

To study fatigue development, three methods of ECAPs' frequency analysis were used:

\section{Discrete wavelet transform (DWT) with Daubechies 5} function

Wavelet decompositions of a signal produce a family of hierarchically organized decompositions situated in different frequency bands. Different wavelet transforms are generated from a basic function (mother wavelet function), by scaling and translating. In DWT, scales and positions are based on power of two called dyadic scales and positions (Daubechies 1992; Wang et al. 2004). DWT application on a signal at its first step produces two sets of coefficients - approximation and detail coefficients. These coefficients are the result of low pass filter application for approximation and high pass filter for detailisation of frequency components of the initial signal. These filters are constructed on the basis of one scaling function and a set of wavelet functions, whose number is equal to the depth of the decomposition tree (5 in our case). The application of these filters splits the signal frequency content into two halves on each step of the decomposition procedure (quadrature mirror filters). The frequency bands were calculated according to the centre frequency of the wavelets applied and to the maximum frequency contents of the potential (Abry 1977; Daubechies 1992). The Daubechies 5 wavelet function, asymmetrical like most other wavelet functions from this family, was chosen on the account of best "fitting" to the analyzed ECAPs. The proof that the best fitting is chosen is the found good correlation $\left(\mathrm{R}^{2}=0.851\right)$ between the shape of the action potentials and Daubechies 5 wavelet function. The $\mathrm{R}^{2}$ value is known as a coefficient of determination. The algorithm of applied wavelet function is numerical with polynomial approximation. DWT (Daubechies 5 function) was applied with MatLab package software.

As the wavelet transforms themselves suffer from apparent lack of quantitative results (as it involves a transform from onedimensional time series to a two-dimensional time-frequency image) we performed root mean square (RMS) analysis of these coefficients in each scale (frequency band respectively).

2. Fast Fourier transform (FFT) - calculation of MDF The same set of potentials was used for calculating the MDF with a custom-written script developed in SPIKE 2 (CED,
Cambridge, UK). MDF of the ECAPs was calculated from power spectral density functions obtained by FFT. The normalized values of $\mathrm{MDF}(\triangle \mathrm{MDF}[\%])$ were calculated as $\Delta \mathrm{T}_{1}[\%]$.

\section{Spectral indices}

Four different spectral indices were applied. They were calculated by the conventional FFT algorithm based on EMG spectral characteristics in frequency domain. They have been constructed as the ratio between the signal spectral moment of order 1 and normalizing spectral moments of order $k=$ $2,3,4,5$. Spectral moments represent the area under the spectral curve after multiplication by the frequency raised to the power of $k$ (called order $k$ of the moment) as the weighting function.

These indices differentions in their normalizing spectral moment $\left(F I_{m m s k}\right)$ order $k$. They were calculated as follows:

$$
F I_{n m s k}=\frac{\int_{f_{1}}^{f_{2}} f^{-l} \cdot P S(f) \cdot d f}{\int_{f_{1}}^{f_{2}} f^{k} \cdot P S(f) \cdot d f}
$$

where $P S(f)$ is spectral power for current frequency $f ; k$ is 2 , 3,4 or $5 ; f_{1}$ and $f_{2}$ stand for the boundary frequencies (lowest and highest) of the power spectrum. To follow dynamics of fatigue, the changes in spectral indices were normalized to the value characterizing the initial period of the uninterrupted fibre activity.

\section{Correlation analysis between $\triangle R M S[\%]$ and $\Delta T_{1}[\%]$}

The correlation analysis between $\Delta \mathrm{RMS}[\%]$ values of the wavelet coefficients and $\Delta \mathrm{T}_{1}[\%]$ was assessed according to RMS values in scale 1 because the frequency content of $T_{1}$ corresponds to the frequencies of that scale (see Fig. 1)

Nonparametric local polynomial regression analysis (first-order fit) was performed using Statistica 6.0 package software. The regression lines were calculated between $\Delta T_{1}$ and $\triangle \mathrm{RMS}$ coefficients for both fibre types and experimental protocols in order to study the relationship between $\mathrm{T}_{1}$ changes and changes in the tested RMS coefficients in frequency scale 1 .

The values from the different spectral methods are calculated and presented on the graphs at intervals of every $25 \%$ of ET for each fibre.

\section{Statistics}

Data are plotted as mean \pm standard errors of the mean (SEM) at every $25 \%$ of ET for each fibre (for SMFs: $n=15$; for FMSs: $n=12$ in each group - sham and MMW exposed fibres, respectively). Kolmogorov-Smirnov test (one-sample K-S test) was used to define, whether the distribution of the parameters analyzed is normal. Data were non-normally distributed. 


\section{SMF}

\section{FMF}

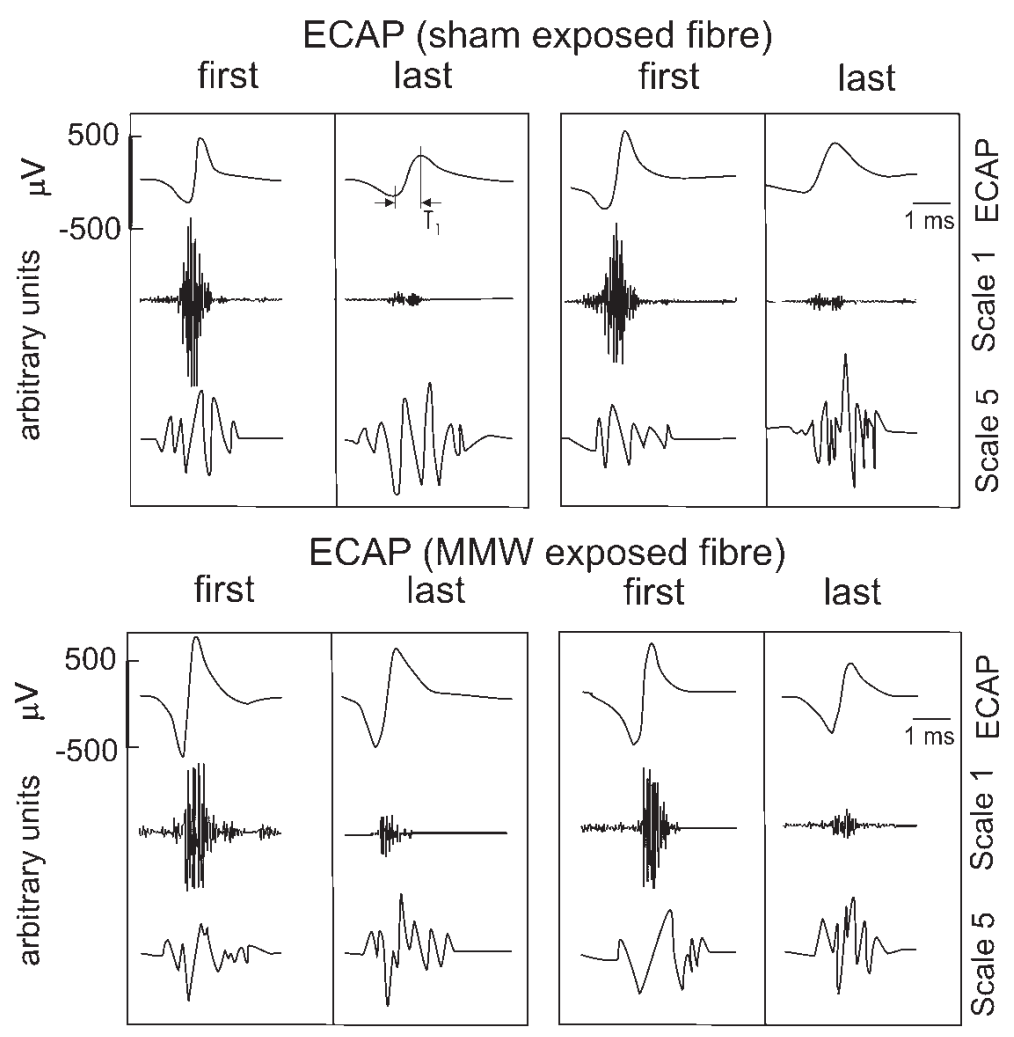

Figure 1. First and last ECAPs during the uninterrupted activity in both experimental protocols (sham exposure and MMW exposure) of SMF and FMF (top rows) with designation of $\mathrm{T}_{1}$ time interval and their averaged wavelet coefficients in time-frequency domain in high (scale 1 from 985 to $1977 \mathrm{~Hz}$ ) and low (scale 5 from 61.781 to $122.562 \mathrm{~Hz}$ ) frequencies (middle and low rows, respectively).

Hence we applied non-parametric one-way Kruskal-Wallis analysis of variance to estimate the significance level of differences between the changes in the parameters of ECAPs recorded from exposed to MMW field and sham exposed muscle fibres as well as between those from SMF and FMF during their uninterrupted activity. The software packages for K-S test were SPSS, and for Kruskal-Wallis - user-defined script in MATLAB. By Kruskal-Wallis analysis the statistical significance of differences at current time point $v$ s. previous one of $\triangle \mathrm{RMS}, \triangle \mathrm{MDF}$ and spectral indices was assessed.

\section{Results}

\section{Wavelet analysis}

Samples of first and last ECAPs from four trials of both experimental protocols (uninterrupted activity of MMW exposed and sham exposed slow- and fast-fatigable fibres) as well as the correspondent high-frequency scale 1 and low-frequency scale 5 (between 988.5 to $1977 \mathrm{~Hz}$ and from
61.781 to $122.562 \mathrm{~Hz}$, respectively) are represented in Fig. 1. The graphic representation of the calculated wavelet coefficients (Fig. 1, middle rows) illustrates a lowering of high frequency content, which correspond to an increase of potential time parameters (especially to the well-defined $\mathrm{T}_{1}$ phase, designated on the Figure). The bottom rows of Fig. 1 show a compression towards the low frequency content of the last potentials in all four panels. The averaged wavelet coefficients are calculated for all DWT analyzed potentials ( $n=15$ for SMFs and $n=12$ for FMFs for each group - sham exposed and MMW exposed fibres). The changes are more pronounced in ECAPs from FMFs compared to those from SMFs as well as in ECAPs from sham exposed fibres $v s$. MMW exposed muscle fibres which is more evident in Fig. 2. The values of RMS analysis of ECAPs wavelet coefficients calculated from sham exposed and MMW exposed SMFs and FMFs recorded during uninterrupted activity (accepted as $100 \%$ ET of each fibre) are presented as $\Delta$ RMS [\%] in Fig. 2. The wavelet coefficients are calculated in high frequency scale 1 and low frequency scale 5 . A statistically significant decrease of $\triangle \mathrm{RMS}$ in the high frequencies (scale 1) as well as 


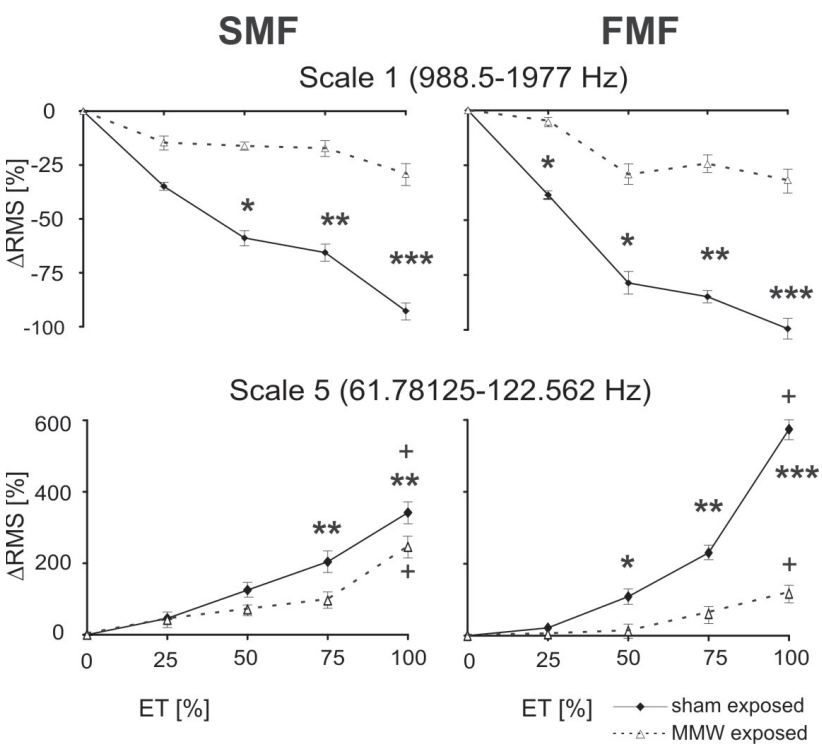

Figure 2. Changes in averaged and normalized values, calculated by RMS analysis of ECAPs wavelet coefficients during uninterrupted activity (ET 100\% divided into four equal time intervals). $n=15$ for sham exposed and MMW exposed SMFs and $n=12$ for sham exposed and MMW exposed FMFs. Symbols of asterisk indicate statistically significant differences between values of the potentials from sham exposed and MMW exposed fibres in the corresponding scales 1 and $5 ;$ symbol $^{+}$indicates statistically significant difference between values of SMF and FMF potentials in low frequency scale 5 . ${ }^{+, *} p<0.05 ;{ }^{* *} p<0.01 ;{ }^{* * *} p<0.001$.

an increase of the low frequencies (scale 5) is evidenced. In scale 1 for both fibre types, the percentage decreases in $\triangle \mathrm{RMS}$ were more pronounced in the potentials from sham exposed fibres vs. MMW exposed ones $(91.35 \pm 3.85 \%$ vs. $29.81 \pm$ $6.73 \%$ for SMFs and $101.53 \pm 5.38 \%$ vs. $32.69 \pm 5.58 \%$ for FMFs). These changes were also fibre type-dependent - more pronounced in ECAPs from FMFs compared to those from SMFs. The statistically significant difference between changes in frequency content of potentials from FMFs and SMFs in scale 1 is visible as an earlier appearance (at 25\% of ET) for FMFs vs. those for SMFs (at $50 \%$ of ET). For FMFs' potentials the steeper course of the changes in $\triangle \mathrm{RMS}$ developed from $25 \%$ of muscle fibre activity $v$ s. those of SMFs (from 50\%) (Fig. 2, scale 1). The values of $\triangle \mathrm{RMS}$ in scale 5 increased in sham exposed for both fibre types (for FMFs sharply - by $570.00 \pm 32.28 \%$ vs. $340.38 \pm 43.86 \%$ for SMFs) with later appearance of statistically significant difference between them compared to that in MMW exposed while in high frequency scale 1 it was evident at 75 and 50\% ET for SMFs and FMFs, respectively. A statistically significant difference between values of $\triangle$ RMS changes of ECAPs from sham exposed SMF and FMFs at $100 \%$ ET was found as the changes in FMFs were larger than that in SMFs as usual. But the alterations of $\triangle$ RMS for MMW exposed fibres showed an exception - in SMFs they were larger $(242.28 \pm 8.86 \%)$ than those in FMFs $(92.28 \pm 3.58 \%)$ at the activity end $(100 \%$ ET). This difference between both fibre types was statistically significant (Fig. 2, scale $5, p<0.05$ ) due to their different ET. It was different for FMFs sham exposed and MMW exposed ones the mean ET was $49.5 \mathrm{~s}$ and $66.5 \mathrm{~s}$, respectively and for SMF sham exposed and MMW exposed ones it was $163 \mathrm{~s}$ and $165.21 \mathrm{~s}$, respectively. The time-dependent statistical data showed that for sham exposed SMFs in scale 1 and 5 a statistical significant difference between the values at $50 \%$ vs. $25 \%$ till the end of ET was found. For the MMW exposed ones this statistical significant difference occurs at $100 \%$ vs. $75 \%$ of ET in both scales. For sham exposed FMFs the results were similar to those of SMFs. For MMW exposed FMFs the statistical difference was observed in scale 1 at $50 \%$ ET and in scale 5 at $100 \%$ ET $v s$. values at $75 \%$ of ET.

\section{Correlation between $\triangle R M S$ and $\Delta T_{1}$}

Due to the observed correspondence between the reduction of high frequencies in scale 1 and shape of the ECAPs expressed in delay of $\mathrm{T}_{1}$ (see Fig. 1), we performed a correlation analysis between the changes of $\Delta \mathrm{RMS}$ in scale 1 and $\Delta \mathrm{T}_{1}$. At the end of ET the duration of $\Delta \mathrm{T}_{1}$ increased as follows: for SMFs - sham exposed by $44 \%$ and MMW exposed by $33 \%$, as well as for FMFs - sham exposed by $52 \%$ and MMW exposed by $41 \%$. The correlation was estimated using first-order polynomial fit (local linear regression). The computed values of $\Delta \mathrm{RMS}$ correlated with those of $\Delta \mathrm{T}_{1}$ as follows: for SMFs with $C_{f}=-0.9058$ and $C_{f}=-0.9905$ for MMW exposed and sham exposed, respectively and for
SMF

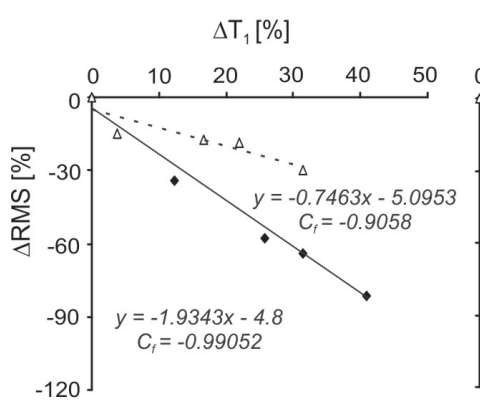

\section{FMF}

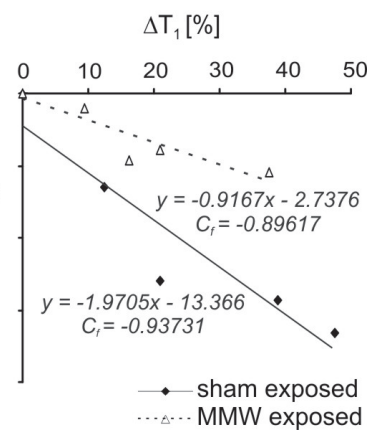

Figure 3. Correlation between the averaged normalized values of RMS wavelet coefficients in high frequency scale 1 and these of the time intervals between ECAPs positive and negative maxima $\left(\Delta \mathrm{T}_{1}[\%]\right)$ of the sham exposed and MMW exposed SMFs and FMFs analyzed in Fig. 2. $y$, the linear equation describing approximating lines; $C_{\mathrm{f}}$, the correlation coefficient and gives the degree of correlation between $\triangle \mathrm{RMS}$ and $\Delta \mathrm{T}_{1}$. 
FMFs with $C_{f}=-0.8961$ and $C_{f}=-0.9373$ for MMW exposed and sham exposed, respectively. The steeper course of the lines presenting the changes in values of $\Delta \mathrm{RMS}$ and $\Delta \mathrm{T}_{1}$ (correlations between $\Delta \mathrm{RMS}$ and $\Delta \mathrm{T}_{1}$ ) in potentials of sham exposed vs. microwave exposed for both fibre types was evident on Fig. 3.

\section{Fast Fourier Transform (FFT) - calculation of MDF}

Larger decreases of calculated on the basis of FFT $\triangle \mathrm{MDF}$ and MDF frequency values were found in sham exposed FMFs and SMFs compared to those found in MMW exposed fibres from both types as the difference between them was statistically significant at 75 and $100 \%$ ET (Fig. 4). Because of the fact that MDF and the spectral indices reflect total spectral changes, they were indicative for considerable power spectrum shift to the lower frequencies during the uninterrupted activity. The absolute values of MDF decreased from $800 \mathrm{~Hz}$ to nearly $200 \mathrm{~Hz}$ for sham exposed and MMW exposed SMFs (Fig. 4, upper, left) and for FMFs from $1000 \mathrm{~Hz}$ to nearly $200 \mathrm{~Hz}$ for sham exposed and to $400 \mathrm{~Hz}$ for MMW exposed (Fig. 4, upper, right). For sham exposed SMFs $\triangle \mathrm{MDF}$ at the end of ET decreased nearly by $70.51 \pm 1.85 \%$ and by $60.00 \pm$ $3.38 \%$ for MMW exposed ones (Fig. 4, bottom, left). The decrease of these values for sham exposed FMFs was $77.85 \pm$ $4.15 \%$ and $63.08 \pm 3.38 \%$ for MMW exposed fibers at the end of ET (Fig. 4 bottom, right). The changes for both fibre type and experimental protocols differed in short range (7.5-10\%). For FMFs the statistically significant differences between $\triangle \mathrm{MDF}$ changes in ECAPs from sham exposed and MMW exposed appeared from 50\% up to $100 \%$ of ET and for SMFs it was visible from $75 \%$ to the end (100\% ET). A statistical significance between $\triangle \mathrm{MDF}$ values through whole ET (at every time point since $25 \%$ till $100 \%$ of ET) was observed only for the sham exposed FMFs.

\section{Spectral indices}

Normalized spectral indices increased during uninterrupted activity of SMFs (Fig. 5A) and FMFs (Fig. 5B).The increment became larger with progress of fatigue (up to the end of ET) that makes the dependences non-linear. Deviation from linearity was more pronounced for sham exposed fibres (solid lines) compared to the MMW exposed ones (dotted lines). The statistically significant differences between changes in spectral indices were found as early as the higher the moment of spectral index is (Index 4 and 5) as well as depending on fibre types (earlier at FMFs - from 50\% ET up to $100 \%$ at Index 4 and 5 while at SMFs - from $75 \%$ up to $100 \%$ ET). The higher the moment of spectral index is, the larger the index value was. Stronger changes for sham exposed (solid lines) vs. MMW exposed fibres (dotted lines) were visible. Like the changes in $\triangle \mathrm{RMS}$ coefficients in low frequency scale,

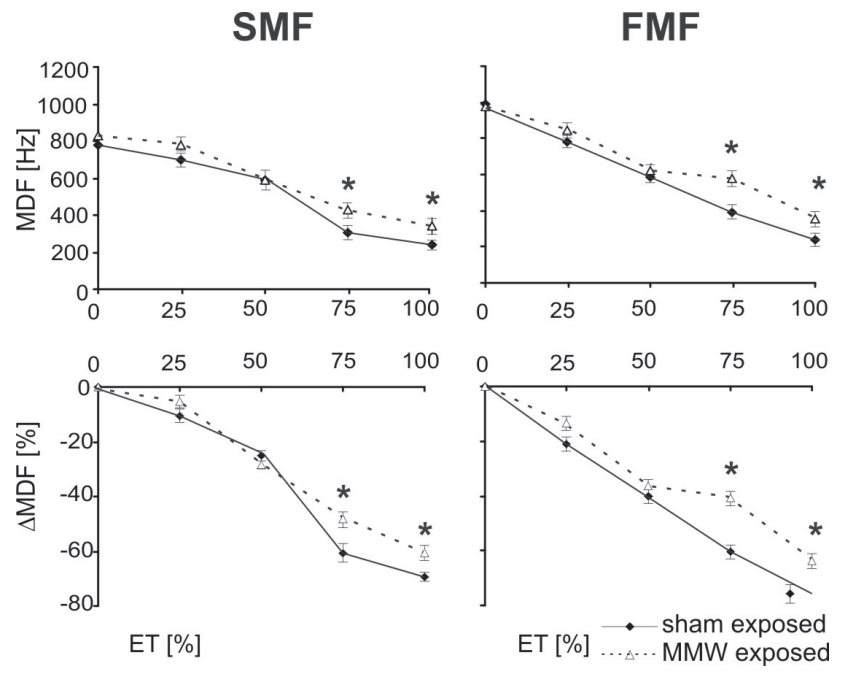

Figure 4. Changes in the averaged MDF (top panels) and normalized changes of MDF of the analyzed potentials (bottom panels). Designations are the same as in Figs. 1 and 2.

higher changes in SMFs MMW exposed vs. those in FMFs with statistically significance in the differences between them were found at index 4 and 5 (Fig. 5, $p<0.05$ ). The statistical significant differences through time for spectral indices of SMFs of both groups were observed at the end of ET (100\% of ET vs. 75\% ET). For sham exposed FMFs statistical significant difference occurs earlier (at 75\% vs. $50 \%$ of ET) in all indices and at 100\% ET for Index 4 and 5. For MMW exposed FMFs statistical significant differences through time exist only in Index 4 and 5 at 75\% ET and in Index 3 at $100 \%$ ET at $p<0.05$ in all analysed parameters of both fibre types and experimental groups.

\section{Discussion}

The present study focuses on fatigue induced changes in potential frequency content and methods for their assessment. We applied a new method for frequency analysis named "Spectral indices" which was successfully utilized in the study of spectral characteristics changes of EMG signals superficially recorded. The obtained results showed that they as well as MDF have good reliability for frequency analysis but the sensitivity of the spectral indices was much more pronounced (Arabadzhiev et al. 2005; Dimitrova et al. 2005, 2009; Dimitrov et al. 2006; Dimitrov 2008). In the present study the spectral indices for the first time were used for processing of potentials from single muscle fibres. For frequency analysis of the same fibre potentials FFT (by MDF as a parameter), and DWT were applied. It is also important to mention that our experimental model for peripheral 


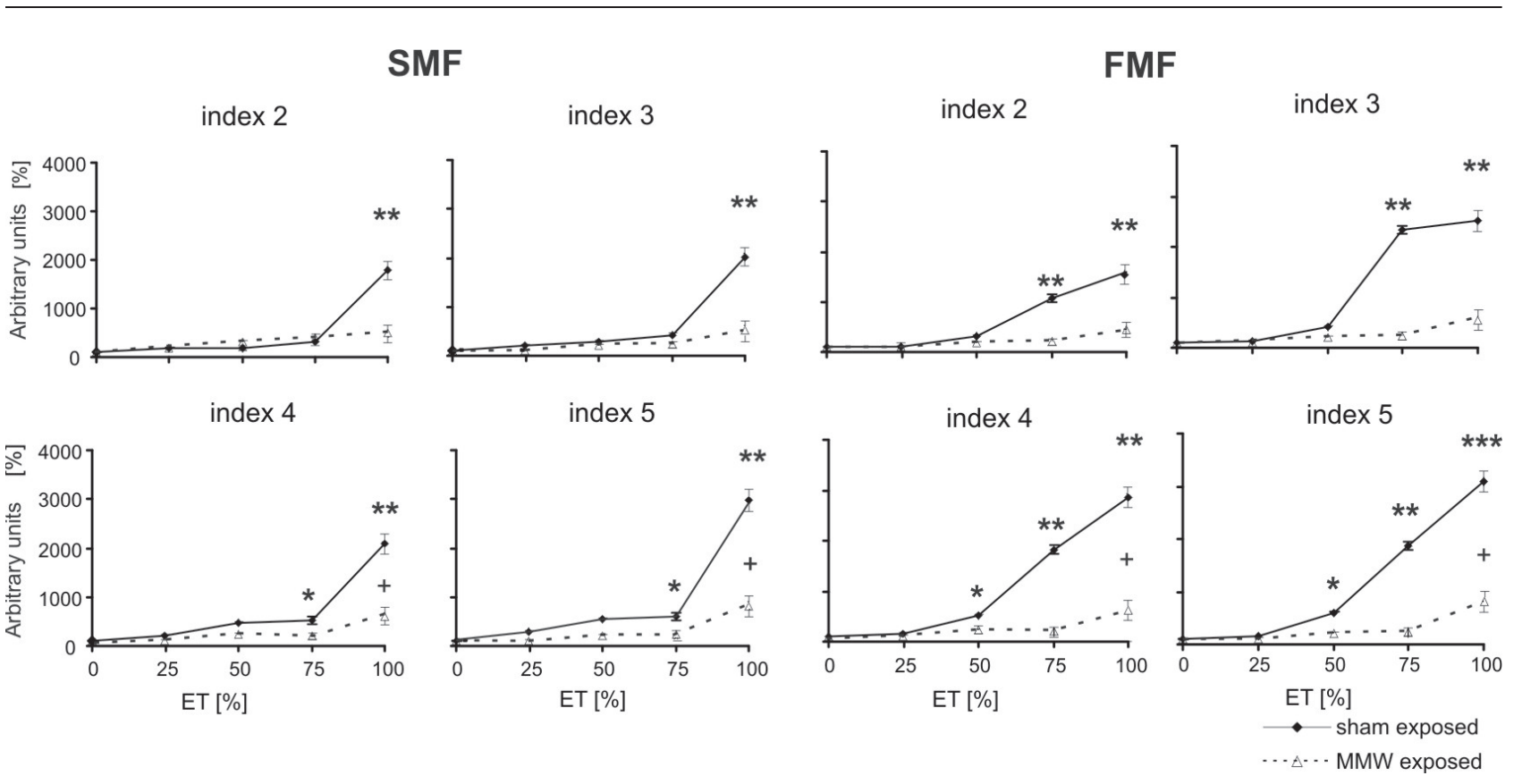

Figure 5. Changes in spectral indices for $k=2,3,4$ and 5 calculated for ECAPs analyzed via the above described methods. Designations are the same as in Figs. 1 and 2.

fatigue is carried out by means of two different approaches for development of fatigue with some distinctions in the stage of induced changes in frequency characteristics of the potentials of MMW exposed and sham exposed isolated fast- and slow-fatigable frog muscle fibres. In earlier investigations fatigue-induced changes in membrane properties and their reflection on the intra- and extracellular action were examined in detail (Radicheva et al. 1994, 1998; Mileva and Radicheva 1996; Vydevska-Chichova et al. 2007). Uninterrupted stimulation of isolated muscle fibres provokes transient changes in the fibre membrane excitability depending on the fibre type as well as on the influence of other factors, such as stimulation frequency, fibre stretch, ionic content of the surrounding medium, electromagnetic irradiation, etc. The diminished membrane excitability predetermines the alterations of action potentials' parameters and in that account for their frequency characteristics (Petrofski and Lind 1980; Stulen and DeLuka 1981; Doud and Walsh 1995; Vydevska-Chichova et al. 2007). The used fatiguing protocol (180-s stimulation with frequency of $5 \mathrm{~Hz}$, temperature of $20-21^{\circ} \mathrm{C}$ of fresh bath solution) develops processes characterizing high frequency fatigue of frog muscle (Westerblad et al. 1991) presented in a specific pattern of muscle fibre activity consisting of alternating periods of uninterrupted activity followed by action potential failure and reappearance (Mileva and Radicheva 1996; Radicheva et al. 1998). Hence, the results concern high frequency fatigue. This means that only membrane processes are affected. The other approach for fatigue development was applied to a preliminary irradia- tion for 60 min on open area of the muscle fibre preparation with continuous MMW field. The results obtained earlier by the same protocol (Radicheva et al. 2000, 2002; Vukova et al. 2008) showed that after MMW exposure the onset of the alterations in the potential parameters were delayed $v s$. those in potentials from sham exposed fibers, i.e. the MMW irradiation provoked some resistance to fatigue. Effects of MMW are related to the dielectric properties of the biomacromolecules and molecular assemblies in the form of functional units such as enzyme complexes, cell membrane receptors, ion channels and skin nerve endings (Bernardi and D'Inzeo 1989). These processes lead to an increase of $\mathrm{Na}^{+}$passive transport or enhance of superficial conductivity, modulation of $\mathrm{Ca}^{2+}$ currents and of the enzyme activity. These are some of the specific mechanisms of the MMW irradiation of skeletal muscle related to the reduced fatigability of MMW exposed fibres and to some distinctions in the stage of induced changes in frequency characteristics of the potentials of MMW exposed and sham exposed isolated fastand slow-fatigable frog muscle fibres. Therefore that allows us to consider which one of the used methods (DWT, MDF based on FFT and Spectral indices) for frequency analysis is the most appropriate to estimate the onset and development of fatigue in single fibres which is the purpose of the present paper. The analyzed excerpts of averaged potentials were sufficient to accomplish this goal.

The shift of EMG power spectrum to lower frequencies during fatigue is well documented (Petrofski and Lind 1980; Stulen and DeLuka 1981; Doud and Walsh 1995). Power 
density distribution of the EMG spectrum obtained from a whole muscle corresponds to the frequency distribution of the action potentials spectrum calculated from the individual muscle fibres of which it is composed (Lindstrom et al. 1970). The power spectrum and its characteristic MDF are reliable tools for neuromuscular activity evaluation (Mannion and Dolan 1994; Kupa et al. 1995; Bonato et al. 2003; Tomazin et al. 2008) as well as for the assessment of peripheral fatigue including in isolated muscle fibres (Vydevska-Chichova et al. 2007). Parallel between results obtained by DWT and FFT (power density) in our preliminary examination of changes in the frequency characteristics of muscle fibres was made (Vukova et al. 2008). Data from RMS analysis of the wavelets indicated that DWT provides a reliable method for evaluation of the onset and progressing of the isolated muscle fibre fatigue. Now the RMS analysis of the wavelet coefficients was calculated in the same way but compared to data from MDF and from the new method of spectral indices. So this approach of comparison seems appropriate to evaluate three methods for analysis in frequency and timefrequency domain for reliability of fatigue-induced changes in EMG signals obtained by needle or wire electrodes, i.e. in potentials from single fibres.

The wavelet analysis allows the changes in the different frequency range as well as the alterations in the time of potential progress in relevant frequency areas to be observed. That permits these changes to be referred to separate phases of the potentials. It was proved by the found correlation dependence between $\Delta \mathrm{RMS}$ and $\Delta \mathrm{T}_{1}$. All these findings essentially inform about the fatigue-affected membrane conductivity. The changes in the wavelets' RMS coefficients in time, during fatigue progress showed in earlier ( $v s . \mathrm{MDF}$ and spectral indices) moments of ET present statistically significant difference between changes in MMW exposed and sham exposed fibres. They were larger for FMFs vs. SMFs as well as larger for sham exposed compared to MMW exposed fibres. Only in scale $5 \Delta$ RMS changes in MMW exposed SMFs at the end of ET (100\%) were larger than that of FMFs with statistically significant differences (Fig. 2). This finding is due to the different duration of ET of both fibre types (mean ET of SMFs was almost three times longer than those of FMFs) assessed at $100 \%$ ET at the end of uninterrupted activity of each fibre. The time-dependent statistically significant difference between $\triangle \mathrm{RMS}$ at the point (moment) of ET compared to that of the value in the preceding time point was found from the beginning of the trial (at 50\% ET) at sham exposed SMFs and FMFs. This result could be explained with the larger changes in sham exposed fibres. The earlier appearance of the signified differences between processed parameters of sham exposed and MMW exposed fibres were accepted as a criterion for sensitivity of the applied methods to the fatigue-induced changes in ECAPs.
Statistically significant differences between changes in MDF and $\triangle \mathrm{MDF}$ of the potentials from MMW exposed and sham exposed fibres was observed only at $75 \%$, and $100 \%$ ET in each group were smaller (in the range of $10-14 \%$ ) than those observed in $\triangle \mathrm{RMS}$ and spectral indices. This fact could be accepted as lighter sensitivity of this method (MDF) to changes compared to that of the other two methods. The rapid linear decrease of $\triangle \mathrm{MDF}$ explains the time-dependent statistically significant differences between $\triangle M D F$ values at a given time point and the value at the preceding one from the beginning of the trial for sham exposed FMFs only.

The spectral indices as well as MDF are calculated on the whole frequency spectrum of the potentials. The first ones give numerical majority of the low frequencies by a raise of coefficient $k$ to the corresponding degree. That is why for $\mathrm{M}$-wave and EMG signals obtained by superficial electrodes they are quite informative. In the present study a great statistically significant increase in the changes of the single potentials' frequency content was measured as late as the second half of ET when the coefficient $k$ is highest. This concerns also time-dependent difference of the values between time points in ET. At indices 4 and 5, statistically significant differences between changes of MMW exposed slow- and fast-fatigable fibres as in the excerption found at $\triangle \mathrm{RMS}$ in scale 5 was observed. This finding was not visible in $\triangle M D F$ data in spite of the prolonged ET of SMFs. So, the spectral indices showed strong sensitivity to fatigue effect at its final stages that made their changes considerably non-linear.

\section{Conclusion}

All tested methods of frequency analysis (DWT, MDF based on FFT and spectral indices) of isolated muscle fibres' potentials are applicable. The observed shift of the frequency content of the potentials to the low frequencies during long lasting activity is fibre type- and research protocol-dependent. The onset of changes in frequency characteristics of potentials from exposed to MMW field fibres was delayed $v s$. that of the sham exposed ones. Usually the frequency changes of the sham exposed fibres were stronger expressed than those of MMW exposed ones and was detected at the earliest time point in all fibre groups by DWT.

It could be concluded that DWT is the most sensitive to the fatigue-induced changes in the frequency characteristics compared to the other two (spectral indices and MDF). This ensues from the possibility the potentials to be analyzed in the corresponding frequency scales obtained by the wavelet decomposition.

Our results suggest that DWT is a reliable method for the invasive EMG for studying the earliest changes in frequency characteristics of the single muscle fibre potentials as well as of their correlation with the potential phases. 
Acknowledgement. Special thanks to Ilina Amer (Sofia University, Department of Modern Languages) for linguistic editing of the text of this publication.

\section{References}

Abry P. (1997): Ondelettes et turbulence. Multirésolutions, algorithms de decomposition, invariance d'échelles. Diderot Editeur, Paris

Arabadzhiev T. I., Dimitrov G. V., Dimitrova N. A. (2005): Simulation analysis of the performance of a novel high sensitive spectral index for quantifying $\mathrm{M}$-wave changes during fatigue. J. Electromyogr. Kinesiol. 15, 149-158; doi:10.1016/j.jelekin.2004.08.003

Arabadzhiev T. I., Dimitrov G. V., Chakarov V. E., Dimitrov A. G., Dimitrova N. A. (2008): Effects of changes in intracellular action potential on potentials recorded by single-fiber, macro, and belly-tendon electrodes. Muscle Nerve 37, 700-712; doi:10.1002/mus.21024

Beck T. W., Housh T. J., Johnson G. O., Weir J. P., Cramer J. T., Coburn J. W., Malek M. H. (2005): Comparison of Fourier and wavelet transform produces for examining the mechanomyographic and electromyographic frequency domain responses during fatiguing isokinetic muscle actions of the biceps brachii. J. Electromyogr. Kinesiol. 15, 190-199; doi:10.1016/j.jelekin.2004.08.007

Bernardi P., D'Inzeo G. (1989): Interaction mechanisms at microscopic level. In: Electromagnetic Biointeraction: Mechanisms, Safety Standards, Protection Guide. (Eds. G. Franceschetti, O. P. Gandhi and M. Grandolfo), pp. 27-57, Plenum Press, New York

Bigland-Ritchie B., Jones D. A., Hosking G. P., Edwards R. H. (1978): Central and peripheral fatigue in sustained maximum voluntary contractions of human quadriceps muscle. Clin. Sci. Mol. Med. 54, 609-614

Bonato P., Ebenbichler G. R., Roy S. H., Lehr S., Posch M., Kollimitzer J., Della Croce U. (2003): Muscle fatigue and fatigue-related biomechanical changes during a cyclic lifting task. Spine 28, 1810-1820; doi:10.1097/01. BRS.0000087500.70575.45

Colliander E. B., Dudley G. A., Tesch P. A. (1988): Skeletal muscle fiber type composition and performance during repeated bouts of maximal concentric contractions. Eur. J. Appl. Physiol. Occup. Physiol. 58, 81-86; doi:10.1007/ BF00636607

Coorevits P., Danneels L., Cambier D., Ramon H., Druyts H., Karlsson J. S., De Moor G., Vanderstraeten G. (2008): Test-retest reliability of wavelet - and Fourier based EMG (instantaneous) median frequencies in the evaluation of back and hip muscle fatigue during isometric back extensions. J. Electromyogr. Kinesiol. 18, 798-806; doi:10.1016/j.jelekin.2007.01.007

Daubechies I. (1992): Ten Lectures on Wavelets. Society for Industrial and Applied Mathematics. Philadelphia

Dimitrov G. V., Arabadzhiev T. I., Mileva K. N., Bowtell J. L., Crichton N., Dimitrova N. A. (2006): Muscle fatigue during dynamic contractions assessed by new spectral indices.
Med. Sci. Sports Exerc. 38, 1971-1979; doi:10.1249/01. mss.0000233794.31659.6d

Dimitrov V. (2008): New approach for assessment of peripheral muscle fatigue. In: Scientific Research Journal of SouthWest University. Proc. 9th Congress of Bulg. Physiol. Society, Bachinovo-Blagoevgrad, November 9-11, 2007. pp. 23-26, ISSN 1313-4558

Dimitrova N. A., Hogrel J. Y., Arabadzhiev T. I., Dimitrov G. V. (2005): Estimate of M-wave changes in human biceps brachii during continuous stimulation. J. Electromyogr. Kinesiol. 15, 341-348; doi:10.1016/j.jelekin.2005.01.005

Dimitrova N. A., Dimitrov G. V. (2006): Electromyography (EMG) modeling. In: Wiley Encyclopedia of Biomedical Engineering. (Ed. A. Metin), John Wiley and Sons, Hoboken, NJ; doi:10.1002/9780471740360.ebs0656

Dimitrova N. A., Arabadzhiev T. I., Hogrel J. Y., Dimitrov G. V. (2009): Fatigue analysis of interference EMG signals obtained from biceps brachii during isometric voluntary contraction at various force levels. J. Electromyogr. Kinesiol. 19, 252-258; doi:10.1016/j.jelekin.2007.08.007

Dolan P., Mannion A. F., Adams M. A. (1995): Fatigue of the erector spinae muscles. A quantitative assessment using "frequency banding" of the surface electromyography signal. Spine 20, 149-159; doi:10.1097/00007632-199501150-00005

Doud J. R., Walsh J. M. (1995): Muscle fatigue and muscle length interaction: effect on the EMG frequency components. Electromyogr. Clin. Neurophysiol. 35, 331-339

Eberstein A, Sandow A. (1963): Fatigue mechanisms in muscle fibres. In: The Effect of Use and Disuse on Neuromuscular Functions. (Ed. E. Gutman and P. Hnik), pp. 515-26, Czechoslovak Academy of Sciences, Prague

Froesea T., Hadjiloucasb S., Galyäoc, Beccerab V., Coelhod C. (2006): Comparison of ECG signal classifiers using discrete wavelet transforms. Pattern Recognit. Lett. 27, 393-407; doi:10.1016/j.patrec.2005.09.002

Grabowski W., Lobsiger E. A., Lüttgau Ch. (1972): The effect of repetitive stimulation at low frequencies upon the electrical and mechanical activity of single muscle fibres. Eur. J. Physiol. 334, 222-239; doi:10.1007/BF00626225

Gydikov A., Gerilovsky L., Radicheva N. (1986a): Extracellular potential field of excited isolated frog muscle fibres immersed in a volume conductor. Gen. Physiol. Biophys. $5,125-134$

Gydikov A., Gerilovsky L., Radicheva N., Trayanova N. (1986b): Influence of the muscle fibre end geometry on the extracellular potentials. Biol. Cybern. 54, 1-8; doi:10.1007/ BF00337110

Gydikov A. (1992): Biophysics of the skeletal muscle extracelluklar potentials. Publishing house of Bulgarian Academi of Sciences, Sofia and Kluwer Academic Publishers, Dordrecht-Boston-London

Hostens I., Seghers J., Spaepen A., Ramon H. (2004): Validation of the wavelet spectral estimation technique in Biceps Brachii and Brachioradialis fatigue assessement during prolonged low-level static and dynamic contractions. J. Electromyogr. Kinesiol. 14, 205-215; doi:10.1016/S10506411(03)00101-9 
Jones D. (1981): Muscle fatigue due to changes beyond beyond the neuromuscular junction. In: Human Muscle Fatigue: Physiological Mechanisms. pp. 178-96, Pitman Medical, London

Karlsson S., Yu J., Metin A. (1999): Enhancement of spectral analysis of myoelectric signals during static contractions using wavelet methods. IEEE Trans. Biomed. Eng. 46, 670-684; doi:10.1109/10.764944

Karlsson S., Yu J., Akay M. (2000): Time-frequency analysis of myoelectric signals during dynamic contractions: a comparative study. IEEE Trans. Biomed. Eng. 47, 228-238; doi:10.1109/10.821766

Kupa E. J., Roy S. H., Kandarian S. C., De Luca C. J. (1995): Effects of muscle fiber type and size on EMG median frequency and conduction velocity. J. Appl. Physiol. 79, 23-32

Lindström L., Magnusson R., Petersén I. (1970): Muscular fatigue and action potential conduction velocity changes studied with frequency analysis of EMG signals. Electromyography 10, 341-355

Mallat S. G. (1989): Theory for multy-resolution signal decomposition the wavelet representation. IEEE Trans. Pattern Anal. Mach. Intell. 11, 674-693; doi:10.1109/34.192463

Mannion A. F., Dolan P. (1994): Electromyographic median frequency changes during isometric contraction of the back extensors to fatigue. Spine 19, 1223-1229

Metzger J., Fitts R. (1986): Fatigue from high and low-frequency muscle stimulation: role of the sarcolemma action potentials. Exp. Neurol. 93, 320-333; doi:10.1016/00144886(86)90193-7

Mileva K., Radicheva N. (1996): Stimulation frequency- and lengthdependent time characteristics of muscle fibre continuous activity. In: Proceedings of the VIIIth International Symposium on Motor Control, Borovets, Bulgaria. (Eds. G. N. Gantchev, V. S. Gurfinkel, D. Stuart, M. Wiesendanger and S. Mori), pp. 207-211, Academic Publishing House "Prof. Marin Drinov", Sofia

Mileva K., Radicheva N., Vydevska M. (1998): Stretch- and simulation frequency-induced changes in extracellular action potentials of muscle fibers during continuous activity. J. Muscle Res. Cell. Motil. 19, 95-103

Morlet D., Peyrin F., Desseigne P., Toubul P., Rubel P. (1993): Wavelet analysis of high-resolution signal averaged ECG's in postinfarction patients. J. Electrocardiol. 26, 311-320; doi:10.1016/0022-0736(93)90052-F

Moxham J., Edwards R. H., Aubier M., De Troyer A., Farkas G., Macklem P. T., Roussos C. (1982): Changes in EMG power spectrum (high-to-low ratio) with force fatigue in humans. J. Appl. Physiol. 53, 1094-1099

Petrofsky J. S., Lind A. R. (1980): The influence of temperature on the amplitude and frequency components of the EMG during brief and sustained isometric contractions. Eur. J. Appl. Physiol. Occup. Physiol. 44, 189-200; doi:10.1007/ BF00421098

Pope M. H., Aleksiev A., Panagiotacopulos N. D., Lee J. S., Wilder D. G., Freisen K., Stielan W., Goel V. K. (2000): Evaluation of low back muscle surface EMG signals using wavelets. Clin. Biomech. 15, 567-573; doi:10.1016/S02680033(00)00024-3
Radicheva N. (1986a): Intracellular and extracellular action potentials in frog muscle fibre upon blocking the potassium conductivity. Acta Physiol. Pharmacol. Bulg. 12, 35-40

Radicheva N. (1986b): Effect of the blocked inactivation of sodium channels on intracellular and extracellular action potentials from isolated frog muscle fibres. Acta Physiol. Pharmacol. Bulg. 12, 27-31

Radicheva N., Gerilovsky L., Gydikov A. (1986): Changes in the muscle fibre extracellular potentials at long-lasting (fatiguing) activity. Eur. J. Appl. Physiol. 55, 545-552; doi:10.1007/BF00421651

Radicheva N., Kolev V. B. (1992): Changes in the muscle fiber extracellular action potentials induced by 4 -aminopiridine. Compt. Rend. Acad. Bulg. Sci. 45, 89-92

Radicheva N., Kolev V., Peneva N. (1993): Influence of intracellular potential and conduction velocity on extracellular muscle fibre potential. J. Electromyogr. Kinesiol. 3, 95-102; doi:10.1016/1050-6411(93)90004-G

Radicheva N., Vydevska M., Mileva K. (1994): Stimulation frequency rate- and stretch-dependent changes in the electrical activity of slow and fast muscle fibres. Compt. Rend. Acad. Bulg. Sci. 47, 89-93

Radicheva N., Mileva K., Vydevska M. (1998): Pattern of continuous muscle fiber activity depending on fiber stretch and stimulation frequency. J. Muscle Res. Cell Motil. 19, 87-94

Radicheva, N.; Mileva, K.; Stojanova, N.; Kristev, I.; Georgieva, B. (2000): Extracellular Ca2+ Removal and Muscle Fibre Activity during Repetitive Stimulation. Compt. Rend. Acad. Bulg. Sci. 53, 101-104

Radicheva N. K., Mileva K., Georgieva B., Kristev I. (2001): Long-lasting (fatiguing activity of isolated muscle fibres influenced by microwave electromagnetic field. Proceedings of IX international Symposium on Motor Control, Varna, 8-12 October, 2000. Acta Physiol. Pharmacol. Bulg. 26, 37-40

Radicheva N., Mileva K., Vukova T., Georgieva B., Kristev I. (2002): Effect of microwave electromagnetic field on skeletal muscle fibre activity. Arch. Physiol. Biochem. 110, 203-214; doi:10.1076/apab.110.3.203.8290

Slavcheva G., Kolev V., Radicheva N. (1996): Extracellular action potentials of skeletal muscle fibre affected by 4 -aminopyridine: a model study. Biol. Cybern. 74, 235-241; doi:10.1007/BF00652224

Sprato P. J., Parnianpour M., Barria E. A., Jagadeesh J. M. (1999): Wavelet analysis of electromyography for back muscle fatigue detection during isokinetic constant-torque exertions. Spine 24, 1791-1798; doi:10.1097/00007632199909010-00008

Sparto P. J., Parnianpour M., Barria E. A., Jagadeesh J. M. (2000): Wavelet and short-time Fourier transform analysis of electromyography for detection of back muscle fatigue. IEEE Trans. Rehabil. Eng. 8, 433-436; doi:10.1109/86.867887

Stulen F. B., DeLuca C. J. (1981): Frequency Parameters of the Myoelectric Signal as a Measure of Muscle Conduction Velocity. IEEE Trans. Biomed. Eng. BME 28, 515-523; doi:10.1109/TBME.1981.324738

Subasi A., Alkan A., Kaklukaya E., Kiynik M. (2005): Wavelet neural network classification of EEG signals by using AR 
model with MLE processing. Neural Netw. 21, 985-997; doi:10.1016/j.neunet.2005.01.006

Tomazin K., Sarabon N., Strojnik V. (2008): Myoelectric alterations after voluntary induced high-and low-frequency fatigue. Journal of Sports science and Medicine 7, 242-248

Torrence C., Compo G. P. (1998): A practical guide to wavelet analysis. Bull. Am. Meteor. Soc. 79, 61-78; doi:10.1175/15200477(1998)079<0061:APGTWA >2.0.CO;2

Vøllestad N. K. (1997): Measurement of human muscle fatigue. J. Neurosci. Methods 74, 219-227; doi:10.1016/S01650270(97)02251-6

Vukova T., Vydevska-Chichova M., Radicheva N. (2008): Fatigue induced changes in muscle fiber action potentials estimated by wavelet analysis. J. Electromyogr. Kinesiol. 18, 397-409; doi:10.1016/j.jelekin.2006.09.014

Vydevska-Chichova M., Mileva K., Radicheva N. (2007): Differential changes in myoelectric characteristics of slow and fast fatigable muscle fibres during long lasting activity. J.
Electromyogr. Kinesiol. 17, 131-141; doi:10.1016/j.jelekin.2006.01.004

Wang S., Xuguang L., Yianni J., Aziz T., Stein J. (2004): Extractiong burst and tonic components from surface electromyograms in dystonia using adaptive wavelet shrinkage. J. Neurosci. Methods 139, 177-184; doi:10.1016/j.jneumeth.2004.04.024

Warren G. L., Ingalls C. P., Shah S. J., Armstrong R. B. (1999): Uncoupling of in vivo torque production from EMG in mouse muscles injured by eccentric contractions. J. Physiol. 515, 609-619; doi:10.1111/j.14697793.1999.609ac.x

Westerblad H., Lee J., Lannergren J., Allen D. (1991): Cellular mechanisms of fatigue in skeletal muscle. Am. J. Physiol. Cell Physiol. 261, 195-209

Received: December 23, 2009

Final version accepted: April 6, 2010 